Article

\title{
Impact of Opioid Analgesia and Inhalation Sedation Kalinox on Pain and Radial Artery Spasm during Transradial Coronary Angiography
}

\author{
Caroline Birgy ${ }^{1}$, Antonin Trimaille ${ }^{1,2} \oplus$, Nathan Messas ${ }^{1}$, Jessica Ristorto ${ }^{1}$, Anas Kayali ${ }^{1}$, \\ Benjamin Marchandot ${ }^{1}$, Thomas Cardi ${ }^{1}$, Sébastien Hess ${ }^{1}{ }^{10}$, Marion Kibler ${ }^{1}$, Laurence Jesel ${ }^{1,2}$, \\ Patrick Ohlmann ${ }^{1}$ and Olivier Morel ${ }^{1,2, *}$ \\ 1 Pôle d'Activité Médico-Chirurgicale Cardiovasculaire, Hôpitaux Universitaires de Strasbourg \\ Université de Strasbourg, 67000 Strasbourg, France; c.birgy@live.fr (C.B.); \\ antonin.trimaille@chru-strasbourg.fr (A.T.); haimmessas@hotmail.fr (N.M.); ristorto-jess@hotmail.fr (J.R.); \\ anas.kayali@chru-strasbourg.fr (A.K.); benjamin.marchandot@gmail.com (B.M.); \\ thomas.cardi@chru-strasbourg.fr (T.C.); sebastien.hess@chru-strasbourg.fr (S.H.); \\ marion.kibler@chru-strasbourg.fr (M.K.); laurence.jesel@chru-strasbourg.fr (L.J.); \\ patrick.ohlmann@chru-strasbourg.fr (P.O.) \\ 2 INSERM UMR-1260 Regenerative Nanomedicine, Université de Strasbourg, 67000 Strasbourg, France \\ * Correspondence: olivier.morel@chru-strasbourg.fr; Tel.: +33-369-551-736
}

Received: 19 June 2020; Accepted: 21 August 2020; Published: 25 August 2020

\begin{abstract}
With respect to the transfemoral approach, transradial procedures enable a drastic reduction of bleeding events and are associated with a reduction of mortality. Radial artery spasm (RAS) is one of the most common complications and may lead to patient discomfort and procedural failure. Currently, there is no consensus on the optimal sedation protocol to avoid RAS. The aim of this study was to investigate the respective impact of opioids analgesia and inhalation sedation with a $50 \%$ nitrous oxide/oxygen premix (Kalinox) on pain and occurrence of RAS during transradial coronary procedures. Consecutive patients undergoing transradial coronary angiography were prospectively enrolled in one, single center observational study (Nouvel Hôpital Civil, Strasbourg, France). Patients received opioids analgesia or inhalation sedation with Kalinox. The primary endpoints of the study were the incidence of a pain scale $\geq 5 / 10$ and the occurrence of RAS. The secondary endpoints were the incidence of side effects. A total of 325 patients were enrolled (185 in the opioids analgesia group, 140 in the Kalinox group). RAS and pain scale $\geq 5$ rates were not significantly different in the opioids analgesia and Kalinox groups (respectively $13.5 \%$ vs. $10.0 \%$ and $16.2 \%$ vs. $11.4 \%$ ). Headache was more frequently observed in the Kalinox group $(6.4 \%$ vs. $0.0 \% ; p=0.002)$. By multivariate analysis, female gender, BMI $<25 \mathrm{~kg} / \mathrm{m}^{2}$, puncture difficulty, the use of plastic needle and $6 \mathrm{~F}$ sheath were identified as independent predictors of RAS. Procedural inhalation sedation by Kalinox is as safe as opioids analgesia during transradial coronary angiography.
\end{abstract}

Keywords: percutaneous coronary intervention; radial artery spasm; analgesia; MEOPA; Kalinox; Entonox; $\mathrm{N}_{2} \mathrm{O} / \mathrm{O}_{2}$; nitrous oxide sedation

\section{Introduction}

With respect to the transfemoral approach, transradial procedures enable a drastic reduction of bleeding events and are associated with a reduction of mortality [1,2]. Radial artery spasm (RAS) is one of the most common complications and may lead to patient discomfort and procedural failure. Currently, there is no consensus on the optimal sedation protocol to avoid RAS [3,4]. The aim of sedation and analgesia in medical procedures like percutaneous coronary intervention (PCI) is to 
reduce the pain and anxiety caused by the procedure with the use of medications with sedatives, analgesics and amnetics effects.

Opioids are commonly used during PCI but they have numerous side effects including the depression of the respiratory system. Owing to their narrow therapeutic window, restriction of their use has been advocated by regulatory agencies and their administration is restricted to anesthesiologists or skilled, critical care physicians. Another important concern that could preclude the systematic use of opioids analgesia during PCI is the recent description of a limited absorption of $\mathrm{P}_{2} \mathrm{Y}_{12}$ inhibitors by opioids as a possible reduction of gut motility [5].

Kalinox is an odorless gas used for procedural sedation and analgesia, typically administered as a $50-50 \%$ nitrous oxide-oxygen mixture $\left(\mathrm{N}_{2} \mathrm{O} / \mathrm{O}_{2}\right)$ to avoid hypoxemia. It is an effective analgesic/anxiolytic agent causing central nervous system depression and euphoria with little effect on the respiratory system. There are many attractive qualities of Kalinox due to its low solubility in the blood which allows a rapid onset of action in the brain as well as rapid clearance through the lungs shortly after administration. It provides a minor amnestic effect [6]. Kalinox has demonstrated its analgesic efficacy and safety in various procedures associated with mild to moderate pain including minor surgery, emergency transportation, venous cannulation, colonoscopy, dentistry, percutaneous liver biopsy or percutaneous renal biopsy [7-9]. Up to now, the use of Kalinox has not been investigated in coronary interventions with a rarity of data exploring its use during cardioversion [10].

The present study was therefore designed to investigate the feasibility and efficacy on pain and RAS of inhalation sedation by $50 \%$ nitrous oxide/oxygen premix (Kalinox) in comparison with opioid analgesia during transradial procedures. In addition, the secondary objective was to assess the predictors of RAS and pain after PCI.

\section{Methods}

\subsection{Patients}

From September 2016 to February 2017, patients undergoing elective or urgent transradial coronary procedures were prospectively enrolled in a high volume (1560 PCI procedures in 2016) PCI center (Nouvel Hôpital Civil, Strasbourg, France). Patients with contraindications (listed in Appendix A) for the use of Kalinox were excluded. Patients received the current analgesia protocol of our center, i.e., opioids analgesia (alfentanil $200 \mu \mathrm{g}$ or morphine $2 \mathrm{mg}$ intravenous), or inhalation sedation by $50 \%$ nitrous oxide/oxygen premix (Kalinox). Operators were encouraged to treat one of two patients with Kalinox and one of two with opioids analgesia. In case of Kalinox intolerance, administration of opioids was permitted. If required to complete the procedure, additional doses of opioids were allowed in patients who received opioid analgesia. All procedures were performed in rooms equipped with a gas scavenging system to limit the medical personnel exposure to nitrous oxide.

Patients' baseline characteristics were recorded: age, gender, size, weight, history of hyperlipidemia, smoking habits, hypertension, diabetes mellitus, coronary arterial disease, peripheral arterial disease (PAD), chronic kidney disease (CKD), medication and materials used during PCI.

For all patients, a puncture was made with a metallic or plastic 19-gauge needle after local anesthesia with 1-4 mL lidocaine 1\%. Numbers of puncture were recorded. A $7 \mathrm{~cm}, 4-6 \mathrm{~F}$ (hydrophilic coated sheath Radifocus ${ }^{\circledR}$ Introducer II set, Terumo, Tokio, Japan) was used and patients in both groups received 3000 to 6000 units heparin according to body weight, $1 \mathrm{mg}$ of isosorbide dinitrate and $1 \mathrm{mg}$ of nicardipine through the radial sheath immediately after insertion. If required, another administration of nicardipine was added. A 0.038 inch guidewire was used (Boston Scientific, Boston, MA, USA). The procedure time was calculated as the time from local anesthesia to the removal of the sheath. Postprocedural hemostasis was achieved by placing of a wrist clamp device (TR Band, Terumo, Tokio, Japan). 
All participants gave their informed written consent for the recording of their data in an anonymized database before the procedure. The study was approved by the University of Strasbourg bioethics committee (reference number: CE-2020-49). The study protocol conforms to the ethical guidelines of the 1975 Declaration of Helsinki.

\subsection{Clinical Endpoints}

Patients' perceived discomfort was assessed in the evening after the procedure or the day after with a visual analogue scale (VAS) whereby the patient graded the discomfort on a scale of 0 to 10 (0 corresponding to "no discomfort" and 10 to "extreme pain and discomfort"). RAS severity was categorized as none, moderate and severe. Spasm was identified by appearance of marked resistance to the movement of the catheter or inability to further advance catheters with or without accompanying forearm pain.

An RAS risk score was calculated for all patients as recently proposed by Giannopulos and coworkers [11]. It consists of five weighted risk factors for radial artery spasm: body mass index (BMI) less than $25 \mathrm{~kg} / \mathrm{m}^{2}$ (1 point), height less than $170 \mathrm{~cm}$ (1 point), current smoking (2 points), hypertension ( 2 points) and PAD (3 points). A predefined score of 4 or more is related to a high risk of RAS [11].

The primary endpoints of the study were the incidence of a pain scale greater than or equal to 5 and the occurrence of RAS. The secondary endpoint included the occurrence of the most frequent side effects of Kalinox such as nausea, vomiting, dizziness or faintness and headache.

\subsection{Statistical Analysis}

Categorical variables were expressed as counts and percentages. Continuous variables were reported as the mean $+/-$ standard deviation (SD) according to their distribution. Categorical variables were compared with Chi-square tests or Fisher's exact tests. Continuous variables were compared with the use of parametric (ANOVA) or nonparametric Mann-Whitney test as appropriate. To determine predictors of radial spasm and pain, regression analysis was performed. Variables with $p<0.05$ in univariate analysis were entered into a stepwise ascending multivariate analysis. A $p$ value $<0.05$ was considered significant. Since Kalinox was never studied in PCI, the sample size could not be calculated. Based on previous studies on RAS, we estimated that a sample size of 300 patients would be adequate. Calculations were performed using SPSS 17.0 for Windows (SPSS Inc., Chicago, IL, USA).

\section{Results}

\subsection{Patients and Baseline Characteristics}

A total of 325 patients were prospectively enrolled. Among these, 185 patients benefited from opioids analgesia by alfentanil/morphine (OA group) and 140 patients from inhalation sedation by Kalinox (K group). Baseline demographics, clinical, biological and angiographic characteristics of the two groups are described in Tables 1 and 2. A higher rate of diabetic patients could be evidenced in the OA group $(31.4 \%$ vs. $21.4 \% ; p=0.032)$. Conversely, patients in the $\mathrm{K}$ group were more frequently smokers (30\% vs. $15.7 \% ; p=0.002)$ and had a higher rate of peripheral artery disease history $(12.1 \%$ vs. $4.9 \% p=0.017)$. Importantly, RAS risk score was equivalent between the two groups (OA $2.4 \pm 1.6$ vs. K $2.6 \pm 1.7 ; p=0.46$ ). Other characteristics (clinical presentation, extent of coronary artery disease, sheath size, procedural time) were equally balanced. 
Table 1. Baseline demographic clinical and biological characteristics according to antalgic group.

\begin{tabular}{|c|c|c|c|}
\hline Variable & Opioid Analgesia $(n=185)$ & Kalinox $(n=140)$ & $p$ Value \\
\hline Age-years & $66.8 \pm 11.9$ & $63.2 \pm 11.3$ & 0.060 \\
\hline Size $-\mathrm{cm}$ & $169.7 \pm 9.2$ & $171.4 \pm 9.4$ & 0.104 \\
\hline Weight—kg & $80.4 \pm 17.1$ & $81.3 \pm 15.5$ & 0.618 \\
\hline Male- $n(\%)$ & $128(69.2)$ & $110(78.6)$ & 0.059 \\
\hline Diabetes mellitus- $n(\%)$ & $58(31.4)$ & $30(21.4)$ & 0.032 \\
\hline Insulinotherapy $-n(\%)$ & $15(8.2)$ & $9(6.4)$ & 0.557 \\
\hline Hypertension- $n(\%)$ & $105(56.8)$ & $75(53.6)$ & 0.567 \\
\hline Smoking- $n(\%)$ & $93(50.3)$ & $88(62.9)$ & 0.024 \\
\hline Current smoking- $n(\%)$ & $29(15.7)$ & $42(30.0)$ & 0.002 \\
\hline Hyperlipidemia- $n(\%)$ & $95(51.4)$ & $68(48.6)$ & 0.620 \\
\hline Known CAD-n $(\%)$ & $87(47)$ & $63(45)$ & 0.717 \\
\hline $\mathrm{PAD}-n(\%)$ & $9(4.9)$ & $17(12.1)$ & 0.017 \\
\hline RAS score-IU & $2.4 \pm 1.6$ & $2.6 \pm 1.7$ & 0.455 \\
\hline $\mathrm{LVEF}-n(\%)$ & $55.7 \pm 10.3$ & $56.2 \pm 10.8$ & 0.672 \\
\hline$\leq 35 \%$ & $3(1.7)$ & $1(0.8)$ & \\
\hline $36-40 \%$ & $7(4.0)$ & $3(2.3)$ & 0.713 \\
\hline $41-50 \%$ & $17(9.8)$ & $12(9.2)$ & \\
\hline$\geq 50 \%$ & $146(84.4)$ & $115(87.8)$ & \\
\hline $\begin{array}{c}\text { eGFR-mL/min } \\
\text { eGFR- } n(\%)\end{array}$ & $74.9 \pm 17.4$ & $76.4 \pm 17.9$ & 0.437 \\
\hline$<30 \mathrm{~mL} / \mathrm{min}$ & 0 & 0 & \\
\hline 30 to $60 \mathrm{~mL} / \mathrm{min}$ & $30(16.5)$ & $17(12.6)$ & 0.626 \\
\hline 60 to $90 \mathrm{~mL} / \mathrm{min}$ & $82(45.1)$ & $63(46.7)$ & \\
\hline eGFR > $90 \mathrm{~mL} / \mathrm{min}$ & $70(38.5)$ & $55(40.7)$ & \\
\hline
\end{tabular}

Data are presented as mean \pm standard deviation in case of any other indication. CAD, coronary artery disease; eGFR, estimated glomerular filtration; IU, international units; LVEF, left ventricular ejection function; PAD, peripheral artery disease; RAS score: radial artery spasm scores.

Table 2. Characteristics of transradial procedure according to antalgic group.

\begin{tabular}{cccc}
\hline Variable & Opioid Analgesia $(\boldsymbol{n = 1 8 5 )}$ & Kalinox $(\boldsymbol{n = 1 4 0 )}$ & $\boldsymbol{p}$ Value \\
\hline Indication of PCI- $n(\%)$ & & & \\
\hline Heart failure & $9(4.9)$ & $11(7.9)$ & 0.266 \\
Programmed angioplasty & $20(10.8)$ & $11(7.9)$ & 0.369 \\
Post-angioplasty control & $6(3.2)$ & $8(5.7)$ & 0.277 \\
Pre-operative exam & $12(6.5)$ & $9(6.4)$ & 0.983 \\
Silent ischemia & $53(28.6)$ & $36(25.7)$ & 0.557 \\
NSTEMI & $19(10.3)$ & $14(10)$ & 0.936 \\
STEMI & $3(1.7)$ & $2(1.4)$ & 0.732 \\
Stable Angina & $38(20.5)$ & $30(21.4)$ & 0.845 \\
Valvular heart disease & $14(7.6)$ & $11(7.9)$ & 0.923 \\
Dyspnea & $6(3.2)$ & $2(1.4)$ & 0.293 \\
Heart rhythm disorder & $2(1.1)$ & $7(5)$ & 0.033 \\
\hline
\end{tabular}


Table 2. Cont.

\begin{tabular}{|c|c|c|c|}
\hline Variable & Opioid Analgesia $(n=185)$ & Kalinox $(n=140)$ & $p$ Value \\
\hline \multicolumn{4}{|c|}{ Height of radial puncture- $n(\%)$} \\
\hline $4 \mathrm{~F}$ & $3(1.6)$ & $4(2.9)$ & 0.447 \\
\hline $5 \mathrm{~F}$ & $104(56.2)$ & $88(62.9)$ & 0.228 \\
\hline $6 \mathrm{~F}$ & $79(42.7)$ & $48(34.3)$ & 0.124 \\
\hline Iode volume-mL & $76.7 \pm 69.3$ & $86.6 \pm 58.4$ & 0.176 \\
\hline Procedural time- $-\min$ & $23 \pm 17.4$ & $22.5 \pm 17.8$ & 0.798 \\
\hline \multicolumn{4}{|l|}{ Height of introducer $-n(\%)$} \\
\hline $4 \mathrm{~F}$ & $6(3.2)$ & $8(5.7)$ & 0.277 \\
\hline $5 F$ & $110(59.5)$ & $90(64.3)$ & 0.376 \\
\hline $6 \mathrm{~F}$ & $69(37.3)$ & $42(30)$ & 0.170 \\
\hline \multicolumn{4}{|l|}{ Type of introducer-n (\%) } \\
\hline Short & $176(95.1)$ & $136(97.1)$ & 0.487 \\
\hline Long hydrophilic & $9(4.9)$ & $4(2.9)$ & 0.360 \\
\hline \multicolumn{4}{|l|}{ Difficulty of puncture- $n(\%)$} \\
\hline One puncture & $162(87.6)$ & $124(88.6)$ & \\
\hline One to three punctures & $17(9.2)$ & $12(8.6)$ & 0.960 \\
\hline More than three & $6(3.2)$ & $4(2.9)$ & \\
\hline \multicolumn{4}{|l|}{ Kind of needle $-n(\%)$} \\
\hline 19 Gauges & $155(83.8)$ & $124(88.6)$ & 0.220 \\
\hline M Coat & $1(0.5)$ & $1(0.7)$ & 0.843 \\
\hline Plastic cathlon & $29(15.7)$ & $15(10.7)$ & 0.195 \\
\hline \multicolumn{4}{|l|}{ Severity of CAD— $n(\%)$} \\
\hline 1-vessel disease & $75(29.1)$ & $72(28.1)$ & 0.791 \\
\hline 2-vessel disease & $58(22.6)$ & $43(16.8)$ & 0.100 \\
\hline 3-vessel disease & $30(11.7)$ & $30(11.7)$ & 0.997 \\
\hline
\end{tabular}

Data are presented as mean \pm standard deviation in case of any other indication. CAD, coronary artery disease; NSTEMI, non-ST segment elevation myocardial infarction; F, French; PCI, percutaneous coronary intervention; STEMI, ST segment elevation myocardial infarction.

\subsection{Radial Artery Spasm}

The incidence of spasm was $12.0 \%$ (39/325). Similar rates of RAS were observed in the Kalinox group and in the opioid analgesia group $(10.0 \%$ vs. $13.5 \%$; $p=0.334)$ (Figure 1). By univariate analysis, female gender, lower body mass index, duration of procedure, puncture difficulty, $6 \mathrm{~F}$ sheath, the use of plastic cathlon and pain scale $\geq 5$ were associated with RAS. No impact of RAS score or high-risk RAS score on RAS could be evidenced. Conversely, nicardipine administration together with the use of 5F sheaths were associated with lower RAS (Table 3). By multivariate analysis, female gender, BMI $<25 \mathrm{~kg} / \mathrm{m}^{2}$, puncture difficulty, the use of plastic needles and $6 \mathrm{~F}$ sheaths were identified as independent predictors of RAS (Table 4). 


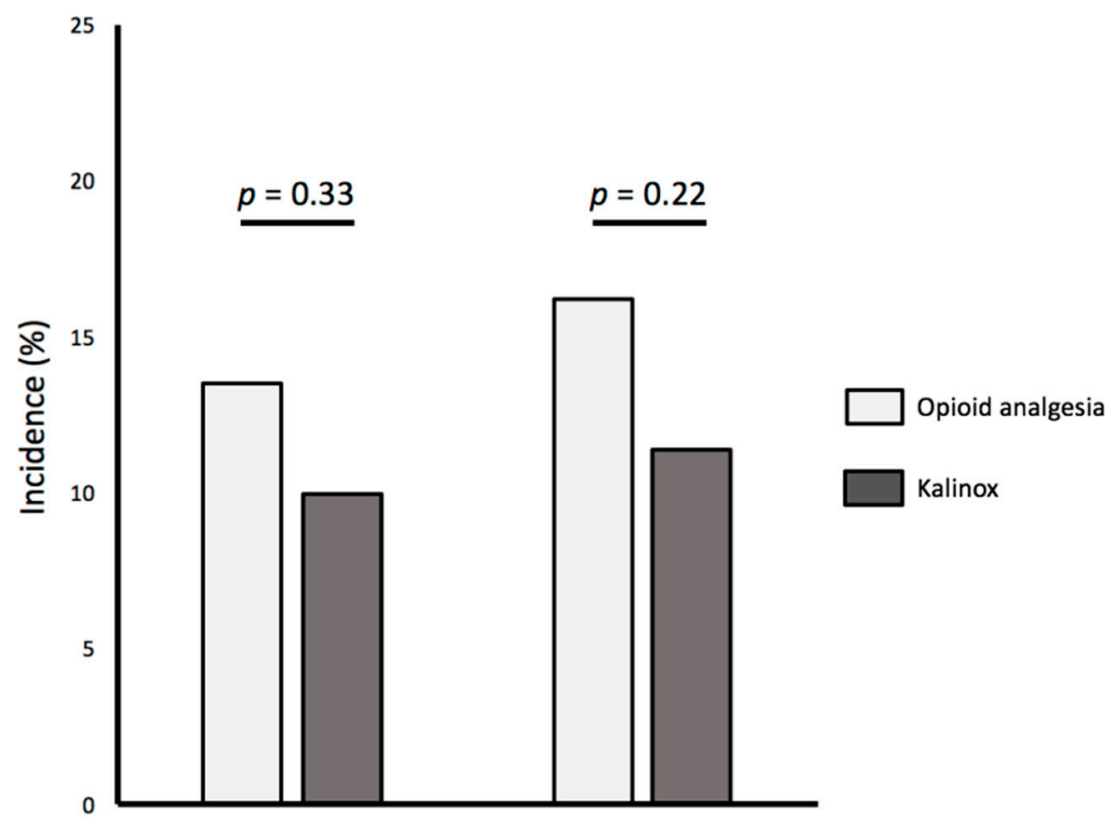

Figure 1. Incidence of Radial Spasm and Pain Scale $\geq 5$ according to antalgic group.

Table 3. Univariate analysis for prediction of Radial Artery Spasm.

\begin{tabular}{|c|c|c|c|}
\hline Variables & HR & CI 95\% & $p$ Value \\
\hline Age $<50$ years & 1.21 & $0.39-3.69$ & 0.734 \\
\hline $\mathrm{BMI}<25 \mathrm{~kg} / \mathrm{m}^{2}$ & 2.09 & $1.06-4.10$ & 0.031 \\
\hline Height $<170 \mathrm{~cm}$ & 1.26 & $0.65-2.45$ & 0.492 \\
\hline Female gender & 3.20 & $1.62-6.30$ & 0.001 \\
\hline Diabetes mellitus & 1.18 & $0.57-2.46$ & 0.640 \\
\hline Insulinotherapy & 0.96 & $0.27-3.39$ & 0.960 \\
\hline Hypertension & 0.97 & $0.50-1.89$ & 0.940 \\
\hline Smoking & 0.97 & $0.50-1.89$ & 0.940 \\
\hline Current smoking & 1.42 & $0.67-3.02$ & $>0.350$ \\
\hline Hyperlipidemia & 1.10 & $0.57-2.14$ & 0.760 \\
\hline Known CAD & 1.87 & $0.95-3.68$ & 0.060 \\
\hline PAD & 1.27 & $0.41-3.88$ & 0.670 \\
\hline LVEF & 1.01 & $0.98-1.04$ & 0.480 \\
\hline CKD & 0.99 & $0.97-1.01$ & 0.330 \\
\hline RAS score & 1.13 & $0.94-1.36$ & 0.192 \\
\hline High risk RAS score & 1.40 & $0.69-2.84$ & 0.339 \\
\hline Procedure duration & 1.018 & $1.002-1.035$ & 0.020 \\
\hline Kalinox use & 0.71 & $0.355-1.424$ & 0.336 \\
\hline \multicolumn{4}{|l|}{ Height of introducer } \\
\hline $5 \mathrm{~F}$ & 0.46 & $0.24-0.91$ & 0.020 \\
\hline $6 \mathrm{~F}$ & 2.62 & $1.33-5.16$ & 0.005 \\
\hline Puncture difficulty & 2.55 & $1.44-4.5$ & 0.001 \\
\hline Supplementary dose of Nicardipine & 0.4 & $0.21-0.75$ & 0.005 \\
\hline Metallic 19 G Needle & 0.31 & $0.14-0.67$ & 0.003 \\
\hline Plastic cathlon & 2.47 & $1.11-5.5$ & 0.020 \\
\hline Pain Scale $\geq 5$ & 3.28 & $1.46-7.38$ & 0.004 \\
\hline
\end{tabular}

CAD, coronary artery disease; CI, confidence interval; CKD, chronic kidney disease; HR, hazard ratio; LVEF, left ventricular ejection function; PAD, peripheral artery disease. 
Table 4. Multivariate analysis for identification of Predictors of Radial Artery Spasm.

\begin{tabular}{cccc}
\hline Variables & HR & CI 95\% & $p$ Value \\
\hline Female gender & 3.15 & $1.45-6.85$ & 0.004 \\
BMI $<25 \mathrm{~kg} / \mathrm{m}^{2}$ & 2.09 & $0.999-4.40$ & 0.050 \\
Plastic cathlon & 2.63 & $1.07-6.46$ & 0.035 \\
6F introducer & 2.89 & $1.23-6.82$ & 0.015 \\
Puncture difficulty & 2.14 & $1.13-4.04$ & 0.019 \\
Pain Scale $\geq 5$ & 2.17 & $0.93-5.07$ & 0.070 \\
Procedure duration & 1.01 & $0.99-1.03$ & 0.300 \\
\hline
\end{tabular}

BMI: body mass index; CI, confidence interval; HR: hazard ratio.

\subsection{Pain Scale}

The incidence of pain scale $\geq 5$ was $14.1 \%$ (46/325). As observed on RAS, similar rates of pain scale $\geq 5$ were observed in the Kalinox group and in the opioid analgesia group $(11.4 \%$ vs. $16.2 \% ; p=0.220)$ (Figure 1). By univariate analysis, female gender, angina and RAS were associated with a pain scale $\geq 5$. No impact of RAS score or high-risk RAS score on pain scale $\geq 5$ could be evidenced (Table 5). By multivariate analysis, angor as an indication of transradial coronary procedure and radial spasm were identified as independent predictors of a pain scale $\geq 5$ (Table 6).

Table 5. Univariate analysis for prediction of Pain Scale $\geq 5$.

\begin{tabular}{cccc}
\hline Variables & HR & CI 95\% & $p$ Value \\
\hline Age $<50$ years & 1.36 & $0.49-3.74$ & 0.550 \\
Weight $<60 \mathrm{~kg}$ & 1.36 & $0.49-3.78$ & 0.550 \\
Female gender & 2.15 & $1.12-4.10$ & 0.020 \\
Diabetes mellitus & 1.07 & $0.53-2.15$ & 0.830 \\
Insulinotherapy & 1.17 & $0.38-3.57$ & 0.780 \\
Hypertension & 0.69 & $0.37-1.30$ & 0.250 \\
Smoking & 0.94 & $0.50-1.77$ & 0.860 \\
Current smoking & 1.15 & $0.55-2.40$ & 0.700 \\
Hyperlipidemia & 1.48 & $0.78-2.79$ & 0.220 \\
Known CAD & 0.87 & $0.46-1.64$ & 0.670 \\
PAD & 0.74 & $0.21-2.57$ & 0.640 \\
LVEF & 1.06 & $0.97-1.03$ & 0.690 \\
CKD & 1.01 & $0.99-1.03$ & 0.210 \\
Angor & 2.33 & $1.18-4.60$ & 0.010 \\
RAS score & 1.10 & $0.92-1.32$ & 0.260 \\
High risk RAS score & 1.40 & $0.69-2.84$ & 0.339 \\
Kalinox use & 0.66 & $0.34-1.27$ & 0.222 \\
Procedure duration & 1.01 & $0.99-1.02$ & 0.230 \\
Introducer 5F & 0.52 & $0.28-0.98$ & 0.040 \\
Spasm & 2.72 & $1.24-5.92$ & 0.012 \\
\hline
\end{tabular}

CAD, coronary artery disease; $\mathrm{CI}$, confidence interval; CKD, chronic kidney disease; HR, hazard ratio; LVEF, left ventricular ejection function; $\mathrm{PAD}$, peripheral artery disease.

Table 6. Multivariate analysis for prediction of Pain Scale $\geq 5$.

\begin{tabular}{cccc}
\hline Variables & HR & CI 95\% & $p$ Value \\
\hline Female gender & 1.81 & $0.92-3.55$ & 0.083 \\
Angor as indication of PCI & 2.22 & $1.11-4.44$ & 0.023 \\
Radial Spasm & 2.28 & $1.01-5.15$ & 0.046 \\
\hline
\end{tabular}

$\mathrm{CI}$, confidence interval; HR, hazard ratio; PCI, percutaneous coronary intervention. 


\subsection{Safety and Side Effects}

Overall, adverse effects were rare. No cases of respiratory depression requiring mechanical support were recorded. In one case, ventilation sedation by Kalinox had to be stopped due to intolerance. Re-administration of opioid to complete the procedure was noticed in 18.9\% (35/185) in the OA group. Administration of Kalinox did not induce more nausea or vomiting (2.9\% versus $1.6 \% ; p=0.44)$. Accordingly, comparable rates of dizziness or faintness could be observed between groups. Conversely, higher rate of headache could be evidenced in patients treated by Kalinox (Table 7).

Table 7. Incidence of adverse effects according to antalgic group.

\begin{tabular}{cccc}
\hline Variables & Opioid Analgesia $(\boldsymbol{n = 1 8 5 )}$ & Kalinox $(n=140)$ & $p$ Value \\
\hline Nausea/vomiting- $n(\%)$ & $3(1.6)$ & $4(2.9)$ & 0.44 \\
Dizziness/faintness— $n(\%)$ & $16(8.6)$ & $7(5)$ & 0.20 \\
Headache- $n(\%)$ & $0(0.0)$ & $9(6.4)$ & 0.002 \\
\hline
\end{tabular}

\section{Discussion}

The main finding of this prospective study is the demonstration of the feasibility of ventilation sedation by Kalinox during transradial coronary procedures. With respect to our current protocol using opioid analgesia, we observed similar incidence of pain and RAS in patients undergoing the transradial approach under Kalinox. Not surprisingly, our findings confirm the deleterious impact of female gender, low body weight, puncture difficulty and large sheath on the occurrence of RAS.

\subsection{Radial Artery Spams Incidence and Risk Factors}

The transradial approach for left heart catheterization and PCI is increasingly used worldwide given its numerous advantages including lower bleeding rates, lower mortality, reduced mobilization time and shorter hospital stays [2]. Increased rates of access site crossover have been described with the radial approach and radial artery spasm has been identified as a major contributor of procedural failure or crossover. Moreover, radial artery spasm may lead to intimal wall injury, intimal thickening and radial occlusion hampering future procedures. The reported frequency of spasm in the transradial approach presents huge variations in the literature ranging from $4 \%$ to higher than $20 \%$ in line with the $12 \%$ reported in the present work $[12,13]$. Numerous factors predisposing to RAS have been identified in the published data including BMI $<25 \mathrm{~kg} / \mathrm{m}^{2}$, height $<170 \mathrm{~cm}$, current smoking, PAD, age, complexity of the procedure, anatomical variations and large radial sheaths and catheter [11]. To allow a better identification of high risk patients, an RAS risk score has been recently proposed by Giannopoulos et al. based on five weighted variables (see methods) with a cut-off of four to predict spasm with a sensitivity of $84.5 \%$ and a specificity of $74.7 \%$ [11]. In the present cohort, we could only identify the noxious impact of BMI $<25 \mathrm{~kg} / \mathrm{m}^{2}$ on RAS but other parameters (current smoker, arterial hypertension, height $<170 \mathrm{~cm}, \mathrm{PAD}$ ) are nonrelevant. Not surprisingly, female gender was identified as an important risk factor of RAS. Important variations in the radial artery diameter between men and women have been described in the past $(2.69 \pm 0.40 \mathrm{~mm}$ in men and $2.43 \pm 0.38 \mathrm{~mm}$ in women) [14] and radial artery-sheath mismatch (ratio between radial artery inner diameter and sheath outer diameter smaller than 1) has been evidenced as the strongest preprocedural predictors of RAS [12]. Of note the 6F radial sheath used in our study has an outer diameter of $2.62 \mathrm{~mm}$ which implies that a mismatch is present in at least $60 \%$ of the women [15].

Noxious connections between anxiety, pain, catecholamines release and RAS have been previously underlined. The possible impact of angina as a clinical presentation is more puzzling. Previous data have suggested that endothelial dysfunction, a key parameter during angina, and characterized by reduced production of nitric oxide (NO) and increased NO breakdown by reactive oxygen species may favor paradoxical vasoconstriction of the vessel and contribute to radial spasm. However, 
recent findings by van der Heiden failed to evidence any link between endothelial function as assessed by EndoPAT and RAS [12].

Along with a technical approach that enables the reduction of spasm such as the downsizing of sheaths and catheters or the use of hydrophilic coated sheaths, the use of appropriate sedation together with vasodilation appears to be an intuitive approach to limit spasm and patient discomfort. In line with this view, data by Deftereos and coworkers have emphasized that routine administration of relatively low doses of an opioid (fentanyl) in combination with benzodiazepine is associated with a significant reduction of spasm, site crossover and patient discomfort [4]. By contrast, another investigation did not evidence any difference in the occurrence of RAS between nitroglycerin alone and nitroglycerin plus midazolam when hydrophilic small sheaths were used [13].

\subsection{Kalinox Effects}

With respect to opioid analgesia, inhalation sedation by $50 \%$ nitrous oxide/oxygen premix (Kalinox) presents several advantages: (i) its administration does not require the presence of an anesthesiologist and can be conducted under the supervision of a trained nurse with conditions and tools of monitoring already used for PCI; (ii) contraindications are limited and allow widespread use (see Appendix A); (iii) its price is affordable. In our experience, procedures could be achieved in all but one patient under Kalinox whereas $18.9 \%$ of the patients in the opioid analgesia required additional administration of opioids. The Kalinox side effect profile was evaluated versus placebo in a previous study in children [16]. A similar side effect profile in both groups was found with only two patients under Kalinox who reported dizziness requiring treatment withdrawal.

The analgesic properties of nitrous oxide $\left(\mathrm{N}_{2} \mathrm{O}\right)$ have been described for more than 150 years and were first exploited in dental surgery, obstetrics and more recently pediatric settings [6]. Its impact on the cardiovascular system remains poorly investigated. Previous findings have suggested that 50/50 mixture of nitrous oxide does not cause significant changes in cardiac output and blood pressure. Although $\mathrm{N}_{2} \mathrm{O}$ has been reported to decrease myocardial contractility in vitro, it also simultaneously stimulates catecholamine release by the sympathetic nervous system hereby leaving cardiac output, blood pressure and heart rate relatively unchanged in vivo [6]. However, the ENIGMA trial (Evaluation of Nitrous Oxide in the Gas Mixture for Anesthesia) [17] sounds the alarm about a possible noxious impact of nitrous oxide on late cardiovascular events. Pathophysiological effects of nitrous oxide on the cardiovascular system may include the inhibition of methionine synthase with resulting hyperhomocysteinemia and endothelial dysfunction [18]. In this trial, the use of nitrous oxide increased the risk of myocardial infarction (OR 1.50, 95\% CI 1.01-2.51; $p=0.04$ ) at long-term follow-up but not at 30 days. However more recently, the ENIGMA-II, a powered study enrolling 7112 non-cardiac-surgery patients at risk of perioperative cardiovascular events, demonstrated the long-term safety of nitrous oxide administration without any elevation of death, myocardial infarction and stroke rates [18]. Altogether, these data support the long-term safety of nitrous oxide administration for the patient. However, operators should watch out for air embolism with a rigorous prevention of air presence in the nitrous oxide circuit. Moreover, the cath lab should be equipped with gas scavenging systems to limit the exposure of medical personnel to nitrous oxide. Chronic exposure to nitrous oxide could reduce fertility and increase the rate of spontaneous abortion in female workers [19].

Other anesthetics without needles have been studied such as lidocaine/prilocaine mixture presented as an ointment which have been shown as an alternative of lidocaine infiltration in transradial cardiac catheterization [20]. However, this anesthetic has never been compared with the $50 \%$ nitrous oxide/oxygen premix (Kalinox).

\subsection{Study Limitations}

The present work is monocentric and has the inherent limitations of any small series. The limited size of the cohort could have hampered the detection of RAS. The study was not randomized. 
Spasm cannot always be defined with objectivity and different definitions have been provided in the past. Angiographic confirmation of the spasm was not performed. In addition, since the PCI operators knew the analgesic regimen of the patient, we cannot exclude a bias in line with the evaluation of RAS. However, angiographic spasm is a common finding (up to $75 \%$ of cases) and there is a weak relation between angiographic spasm and procedural outcomes [21]. The radial artery size was not systematically recorded in this study. Nevertheless, a small radial artery makes the radial artery catheterization difficult and could for this reason increase radial artery spasm. The risk factors included in a high RAS risk score-such as female sex, small patient size or BMI-are related with small radial artery size and could indirectly explain the increase of this complication.

An imbalance in diabetic or smoking status was evidenced between groups. Since current smoking is an important factor of RAS, we could not exclude that the higher rate of smoking in the Kalinox group may have blunted the protective effect of Kalinox on spasm.

Patients evaluated their pain several hours after the procedure which may lead to a reduction in the intensity of the pain actually felt, especially when taken into account that Kalinox has an amnestic effect. Anxiety score was not assessed. Other possible impacts of nitrous oxide on hyperemia such as those induced during fractional flow reserve measurement should be investigated in appropriate trials.

\section{Conclusions}

Procedural inhalation sedation by Kalinox is as safe as opioids analgesia during transradial coronary angiography. Patient-administered $\mathrm{N}_{2} \mathrm{O} / \mathrm{O}_{2}$ inhalation provides a safe and effective analgesia at a reasonable cost with a low rate of side effects. Further studies are needed to definitively recommend Kalinox for routine use during transradial PCI.

Author Contributions: Conceptualization, C.B. and O.M.; methodology, C.B. and O.M.; validation, N.M., L.J., P.O. and O.M.; formal analysis, L.J., P.O. and O.M.; investigation, C.B., J.R., A.K., B.M., T.C., M.K., S.H. and O.M.; data curation, C.B., A.T., J.R., A.K., B.M., T.C. and M.K.; writing-original draft preparation, C.B. and O.M.; writing-review and editing, A.T. and O.M.; supervision, O.M. All authors have read and agreed to the published version of the manuscript.

Funding: This research received no external funding.

Conflicts of Interest: The authors declare no conflict of interest.

\section{Appendix A Contraindications for Use of Kalinox}

- Hypersensitivity to the active substance.

- In patients with signs or symptoms of pneumothorax, pneumopericardium, severe emphysema, gas emboli or head injury.

- Maxillofacial injuries.

- Following deep sea diving with risk of decompression sickness (bubbles of nitrogen).

- Following treatment with heart lung machine or coronary bypass without heart lung machine.

- In patients recently having undergone intraocular injection of gas until the gas in question is fully absorbed, or within 3 months after the last injection of an intraocular gas, because the gas volume may increase in pressure/volume and consequently result in blindness.

- Patients with a severely dilated gastrointestinal tract.

- Following air encephalography.

- During middle ear, inner ear and sinus surgery.

- If air has been injected into the epidural space to determine the placement of the needle for epidural anesthesia.

- In patients showing signs of confusion or in some other way showing signs of increased intracranial pressure.

- Patients with untreated vitamin B12- or folic acid deficiency or diagnosed genetic disorder of the enzyme system involved in metabolism of these vitamins. 
- Patients with a decreased level of consciousness or impaired ability to cooperate and follow instructions due to the risk that further sedation from the nitrous oxide may affect natural protective reflexes.

- Patients with facial injury where use of a face mask may present difficulties or risks.

\section{References}

1. Jolly, S.S.; Yusuf, S.; Cairns, J.; Niemela, K.; Xavier, D.; Widimsky, P.; Budaj, A.; Niemela, M.; Valentin, V.; Lewis, B.S.; et al. Radial versus femoral access for coronary angiography and intervention in patients with acute coronary syndromes (RIVAL): A randomised, parallel group, multicentre trial. Lancet 2011, 377, 1409-1420. [CrossRef]

2. Bavishi, C.; Panwar, S.R.; Dangas, G.D.; Barman, N.; Hasan, C.M.; Baber, U.; Kini, A.S.; Sharma, S.K. Meta-Analysis of Radial Versus Femoral Access for Percutaneous Coronary Interventions in Non-ST-Segment Elevation Acute Coronary Syndrome. Am. J. Cardiol. 2016, 117, 172-178. [CrossRef]

3. Whitehead, N.J.; Clark, A.L.; Williams, T.D.; Collins, N.J.; Boyle, A.J. Sedation and Analgesia for Cardiac Catheterisation and Coronary Intervention. Heart Lung Circ. 2020, 29, 169-177. [CrossRef]

4. Deftereos, S.; Giannopoulos, G.; Raisakis, K.; Hahalis, G.; Kaoukis, A.; Kossyvakis, C.; Avramides, D.; Pappas, L.; Panagopoulou, V.; Pyrgakis, V.; et al. Moderate procedural sedation and opioid analgesia during transradial coronary interventions to prevent spasm: a prospective randomized study. JACC Cardiovasc. Interv. 2013, 6, 267-273. [CrossRef]

5. Kubica, J.; Adamski, P.; Ostrowska, M.; Sikora, J.; Kubica, J.M.; Sroka, W.D.; Stankowska, K.; Buszko, K.; Navarese, E.P.; Jilma, B.; et al. Morphine delays and attenuates ticagrelor exposure and action in patients with myocardial infarction: The randomized, double-blind, placebo-controlled IMPRESSION trial. Eur. Heart J. 2016, 37, 245-252. [CrossRef] [PubMed]

6. Huang, C.; Johnson, N. Nitrous Oxide, From the Operating Room to the Emergency Department. Curr. Emerg. Hosp. Med. Rep. 2016, 4, 11-18. [CrossRef] [PubMed]

7. Galeotti, A.; Garret Bernardin, A.; D'Anto, V.; Ferrazzano, G.F.; Gentile, T.; Viarani, V.; Cassabgi, G.; Cantile, T. Inhalation Conscious Sedation with Nitrous Oxide and Oxygen as Alternative to General Anesthesia in Precooperative, Fearful, and Disabled Pediatric Dental Patients: A Large Survey on 688 Working Sessions. BioMed Res. Int. 2016, 2016, 7289310. [CrossRef]

8. Pietrement, C.; Salomon, R.; Monceaux, F.; Petitjean, C.; Niaudet, P. Analgesia with oxygen-nitrous oxide mixture during percutaneous renal biopsy in children. Arch. Pediatr. 2001, 8, 145-149.

9. Castera, L.; Negre, I.; Samii, K.; Buffet, C. Patient-administered nitrous oxide/oxygen inhalation provides safe and effective analgesia for percutaneous liver biopsy: A randomized placebo-controlled trial $(2,3)$. Am. J. Gastroenterol. 2001, 96, 1553-1557. [CrossRef]

10. Masoumi, K.; Forouzan, A.; Saghari, S.; Feli, M.; Sattari, A.R.; Asgari Darian, A. Sedative and Analgesic Effects of Entonox Gas Compared with Midazolam and Fentanyl in Synchronized Cardioversion. Crit. Care Res. Pract. 2015, 2015, 1-6. [CrossRef] [PubMed]

11. Giannopoulos, G.; Raisakis, K.; Synetos, A.; Davlouros, P.; Hahalis, G.; Alexopoulos, D.; Tousoulis, D.; Lekakis, J.; Stefanadis, C.; Cleman, M.W.; et al. A predictive score of radial artery spasm in patients undergoing transradial percutaneous coronary intervention. Int. J. Cardiol. 2015, 188, 76-80. [CrossRef] [PubMed]

12. Van der Heijden, D.; van Leeuwen, M.A.; Janssens, G.N.; Hermie, J.; Lenzen, M.J.; Ritt, M.J.; van de Ven, P.M.; Kiemeneij, F.; van Royen, N. Endothelial dysfunction and the occurrence of radial artery spasm during transradial coronary procedures: The ACRA-Spasm study. EuroIntervention 2016, 12, 1263-1270. [CrossRef] [PubMed]

13. Astarcioglu, M.A.; Sen, T.; Kilit, C.; Durmus, H.I.; Gozubuyuk, G.; Agus, H.Z.; Kalcik, M.; Karakoyun, S.; Yesin, M.; Dogan, A.; et al. Procedural sedation during transradial coronary angiography to prevent spasm. Herz 2016, 41, 435-438. [CrossRef] [PubMed]

14. Yoo, B.S.; Yoon, J.; Ko, J.Y.; Kim, J.Y.; Lee, S.H.; Hwang, S.O.; Choe, K.H. Anatomical consideration of the radial artery for transradial coronary procedures: Arterial diameter, branching anomaly and vessel tortuosity. Int. J. Cardiol. 2005, 101, 421-427. [CrossRef] [PubMed] 
15. Aminian, A.; Lalmand, J.; Dolatabadi, D. Prevention of radial artery spasm: Importance of a multifactorial approach. JACC Cardiovasc. Interv. 2013, 6, 1214. [CrossRef]

16. Reinoso-Barbero, F.; Pascual-Pascual, S.I.; de Lucas, R.; García, S.; Billoët, C.; Dequenne, V.; Onody, P. Equimolar Nitrous Oxide/Oxygen Versus Placebo for Procedural Pain in Children: A Randomized Trial. Pediatrics 2011, 127, e1464-e1470. [CrossRef]

17. Leslie, K.; Myles, P.S.; Chan, M.T.; Forbes, A.; Paech, M.J.; Peyton, P.; Silbert, B.S.; Williamson, E. Nitrous oxide and long-term morbidity and mortality in the ENIGMA trial. Anesth. Analg. 2011, 112, 387-393. [CrossRef]

18. Leslie, K.; Myles, P.S.; Kasza, J.; Forbes, A.; Peyton, P.J.; Chan, M.T.; Paech, M.J.; Sessler, D.I.; Beattie, W.S.; Devereaux, P.J.; et al. Nitrous Oxide and Serious Long-term Morbidity and Mortality in the Evaluation of Nitrous Oxide in the Gas Mixture for Anaesthesia (ENIGMA)-II Trial. Anesthesiology 2015, 123, 1267-1280. [CrossRef]

19. Rowland, A.S.; Baird, D.D.; Weinberg, C.R.; Shore, D.L.; Shy, C.M.; Wilcox, A.J. Reduced Fertility among Women Employed as Dental Assistants Exposed to High Levels of Nitrous Oxide. N. Engl. J. Med. 1992, 327, 993-997. [CrossRef]

20. Latsios, G.; Toutouzas, K.; Antonopoulos, A.S.; Melidi, E.; Synetos, A.; Vogiatzi, G.; Stasinopoulou, A.; Tsioufis, K.; Tsiamis, E.; Tousoulis, D. Anesthetic ointment only (lidocaine/prilocaine) instead of injectable local lidocaine in trans-radial catheterization: A viable no-needle alternative. J. Interv. Cardiol. 2017, 30, 382-386. [CrossRef]

21. Fukuda, N.; Iwahara, S.; Harada, A.; Yokoyama, S.; Akutsu, K.; Takano, M.; Kobayashi, A.; Kurokawa, S.; Izumi, T. Vasospasms of the radial artery after the transradial approach for coronary angiography and angioplasty. Jpn. Heart J. 2004, 45, 723-731. [CrossRef] [PubMed]

(C) 2020 by the authors. Licensee MDPI, Basel, Switzerland. This article is an open access article distributed under the terms and conditions of the Creative Commons Attribution (CC BY) license (http://creativecommons.org/licenses/by/4.0/). 